\title{
Transcription strategies in terminally differentiated cells: shaken to the core
}

\author{
Katherine A. Jones ${ }^{1}$ \\ Regulatory Biology Laboratory, The Salk Institute for Biological Studies, La Jolla, California 92037, USA
}

The formation of mature tissues and cell types in eukaryotic organisms requires that cells undergo a regulated series of steps leading to terminal cell differentiation, which includes a permanent withdrawal from the cell cycle and the eventual cessation of all cell proliferation. Concomitant with this process is the stable repression of many genes involved in normal cell growth and cell cycle control, accompanied by profound changes in nuclear chromatin structure that arise as previously active genes gradually become silenced and are modified epigenetically to form facultative heterochromatin (Grigoryev et al. 2006). At the same time, newly activated signaling pathways must induce the de novo expression, function, or nuclear localization of the various tissue-specific enhancer-binding proteins needed to regulate the genes responsible for creating and maintaining the differentiated cell phenotype.

A powerful system used to identify the fundamental principles of cell fate specification is the process of skeletal muscle differentation, or myogenesis, in which multipotential mesodermal precursor cells commit and differentiate to a muscle cell fate (Sartorelli and Caretti 2005). This process can be studied in defined sequential stages, wherein the precursor cells first commit to form undifferentiated myoblasts, then differentiate and fuse to form multinucleated myotubes, and subsequently mature into functional myofibers. Key upstream activators in this process include members of the MyoD family of basic helix-loop-helix (bHLH) regulators (MyoD, myogenin, MRF4, and Myf5), as well as the MEF2 family of MADS-box factors (MEF2-A, MEF2-B, MEF2-C, MEF2D). In this system, the myogenic bHLH factors form heterodimers with E-box factors (E12, E47), and function cooperatively with the MEF2 proteins to activate skeletal muscle-specific genes, while also creating strong autoregulatory feedback loops that ensure their own sustained expression (Bassel-Duby and Olson 2006; Baugh and Hunter 2006). MEF2 can also act independently of MyoD through its ability to associate with myocardin and MASTR/SAP domain protein in cardiac and skeletal muscle cells, respectively (Creemers et al. 2006). More-

${ }^{1}$ Correspondence.

E-MAIL jones@salk.edu; FAX (858) 535-8194.

Article is online at http://www.genesdev.org/cgi/doi/10.1101/gad.1598007. over, MEF2C also interacts directly with the Notch coactivator, Mastermind (MamL1), the targeted deletion of which causes a severe muscular dystrophy in mice (Shen et al. 2006). These factors assemble large muscle-specific enhancer complexes and cooperate with other DNAbound activators, such as the serum response factor (SRF), to displace repressors and recruit the coactivators needed to up-regulate transcription (Pipes et al. 2006).

In this issue of Genes \& Development, Deato and Tjian (2007) describe a remarkable new mode of transcriptional regulation in differentiated cells that is centered on the core transcriptional machinery. Detailed analysis of core promoter factor expression in these cells reveals that myotube differentiation is accompanied by a dramatic loss of the TATA-binding protein (TBP) as well as many of the RNA polymerase II (RNAPII) TATA-associated factor (TAF) subunits (TAF1, TAF4, TAF9, TAF10), suggestive of a wholesale extinction of the TFIID complex. The loss of TFIID components, which was detected at both the RNA and protein level, may provide an effective mechanism for large-scale silencing of genes whose functions are needed only in undifferentiated cells, including those that control cell proliferation and progression through the cell cycle. These findings confirm and extend an earlier report from Perletti et al. (2001) that both TBP and TAF4 are down-regulated by targeted proteolysis in $\mathrm{C} 2 \mathrm{C} 12$-derived myotubes and differentiated F9 cells. Deato and Tjian (2007) further discovered that the loss of core factors in differentiated myotubes is highly selective and that the expression of certain other core factors, namely the TATA-related factor TRF3 and the TAF3 protein, persists in differentiated cells. Interestingly, TRF3 and TAF3 were found to interact directly to form a minimal core promoter recognition complex that binds to the Myogenin core promoter and cooperates with the myogenic upstream activators to activate transcription in differentiated myotubes. Further analyses in stable small interfering RNA (siRNA)-expressing cell lines established that TRF3 and TAF3 are both necessary for myogenesis, and have no effect on TFIID function or stability in dividing myoblasts.

Taken together, these findings support a model in which core promoter switching mediates the global repression of TFIID-dependent genes and simultaneously allows for selective activation of myogenic genes that de- 
pend only on the TRF3:TAF3 complex. Whereas differentiation is generally considered to require only the de novo expression of a handful of tissue-specific regulators, the current results show that it can also involve the selective loss of some of the most commonly used RNAPII core transcription factors. This model also challenges the widely held notion that tissue-specific or inducible activators function with a largely static and unchanging core transcription machinery, and raise interesting questions about how the loss of TBP is tolerated at other genes, including the RNAPI and RNAPIII-regulated genes. Additional questions arising concern how the TRF3:TAF3 complex is recruited to myogenic-specific as well as housekeeping genes in differentiated myotubes, whether other variant core factors or chromatin-modifying complexes are also used at these genes, and how these changes in the core transcription machinery might affect the early steps of RNA synthesis and the various histone modifications associated with transcription.

\section{Widespread loss of TFIID subunits in terminally differentiated cells}

These findings draw attention to the still-mysterious inner workings of the core promoter, defined as the region of a gene that spans the RNA start site and nearby regulatory elements that recruit RNAPII to initiate transcription, frequently including the TATA-box, Initiator (Inr), TFIIB recognition element (BRE), or downstream core promoter element (DPE) (Smale and Kadonaga 2003). TBP is a key component of the core machinery required for transcription initiation by all three eukaryotic RNA polymerases (for review, see Thomas and Chiang 2006). TBP is recruited directly to TATA-containing RNAPII genes, but can also be recruited indirectly, through protein-protein interactions to TATA-less genes. A fraction of native TBP resides in multisubunit complexes with various RNA polymerase-specific TAFs, including the RNAPII-specific TFIID and B-TFIID complexes. TBP is also a component of the SNAPc complex, which mediates transcription of U6snRNA and U2snRNA genes, as well as RNAPI-specific (SL1) and RNAPIII-specific (TFIIIB) complexes. The RNAPII-specific complex, TFIID, is recruited with other general initiation factors (TFIIA, TFIIB, TFIIE, TFIIF, TFIIH) to many core promoters upon induction of transcription. Despite its widespread role in transcription, RNAPII transcription proceeds apparently undeterred in $\mathrm{TBP}^{-/-}$knockout mice, which eventually undergo growth arrest and apoptosis at the blastocyst stage (Martianov et al. 2002), indicating that there is at least partial redundancy between TBP and one or more of the developmental stage- or cell-typespecific orthologs (called TRFs) that have been described to date (Thomas and Chiang 2006). In addition, core promoter activity for certain genes can be achieved independently of TBP by TFTC, a TAF-containing complex that is similar to the yeast Spt3-Ada-Gen5-acetyltransferase (SAGA) complex.

The TRFs include the insect-specific TRF1 (Hansen et al. 1997; Holmes and Tjian 2000; Takada et al. 2000), the metazoan TRF2 factor (TBP-like factor) that functions to up-regulate certain zygotic genes early during embryogenesis in worms, frogs, and zebrafish, but not mice (Rabenstein et al. 1999; Teichmann et al. 1999; Veenstra et al. 2000; Zhang et al. 2001; Hochheimer et al. 2002), and the vertebrate-specific TRF3 (also called TBP2 protein) (Persengiev et al. 2003). TRF3/TBP2 is very closely related to TBP within the DNA-binding C-terminal domain (CTD), and diverges more significantly from TBP at the $\mathrm{N}$ terminus of the protein. Like TBP, TRF3 can bind to TATA-box elements, interact with general transcription factors TFIIA and TFIIB, and properly direct transcription initiation in vitro, and has been found to be essential for early embryogenesis in mice, frogs, and zebrafish (Bartfai et al. 2004; Jallow et al. 2004; Xiao et al. 2006; Gazdag et al. 2007). Chromatin immunoprecipitation (ChIP) assays performed in mouse embryonic stem (ES) cells indicate that some genes indiscriminately recruit either TBP or TRF3, or both (including both TATAcontaining and TATA-less genes), whereas other genes preferentially target one or the other of the two proteins, such as the undifferentiated cell-specific Oct4 gene, which specifically recruits TRF3, and not TBP (Yang et al. 2006). Similarly, TRF3 can be selectively recruited to some promoters in Xenopus oocytes, and has been proposed to function in a nonredundant, or only partially redundant manner with TBP during oogenesis and development in frogs (Jallow et al. 2004; Xiao et al. 2006) and in mice (Gazdag et al. 2007).

\section{Selective retention of TRF3 (TRF3/TBP2) and TAF3 proteins in differentiated myotubes}

Given the many reports of coexpression and partial redundancy in function between TBP and TRF3, it is striking that both myoblast and F9 cells undergo a global loss of TBP/TFIID components in post-mitotic differentiated cells. To better detect and quantify this phenomenon in muscle cells, it was necessary for Deato and Tjian (2007) to use highly purified cultures of C2C12-derived myotubes that had been separated away from the so-called "reserve cells," which retain expression of the TBP and the canonical TFIID complex and have not permanently exited the cell cycle. In addition, TBP and TAF expression levels were examined in primary skeletal myoblasts and myofibers. These analyses further established that a relatively poorly characterized TAF subunit, TAF3 (Gangloff et al. 2001), was stably expressed along with TRE3 in differentiated cells. Most interestingly, TAF3 and TRF3 were found to interact directly, both in extracts and as recombinant proteins, to form an alternative core promoter complex. Stable cell lines expressing siRNAs directed to either or both subunits of the TRF3:TAF3 complex strongly blocked differentiation and impaired the expression of myogenic marker genes encoding Myogenin, MyoD, and myosin. Both the expression of these genes as well as proper cell differentiation were rescued upon ectopic expression of the two proteins in the 
C2C12 cell lines, indicating that the TRF3:TAF3 act upstream of, and in conjunction with, many of the tissuespecific regulators present in differentiated myotubes. Interestingly, these studies also revealed that the TRF3:TAF3 complex is required for optimal down-regulation of TBP/TFIID in differentiating cells, and confirmed that the two complexes have nonredundant functions in the nondividing myotubes. Thus, in contrast with the tissue-specific TAF variants (Verrijzer 2001), TAF3 is expressed in both differentiated and undifferentiated cells and appears to have important roles in both cell types.

\section{TRF3 may also be linked to retinoblastoma $(\mathbf{R b})$ expression}

Given these exciting new findings, it is interesting to consider other possible target genes for TRF3 in differentiated cells. As shown by Jallow et al. (2004), antisense knockdown of TRF3/TBP2 in Xenopus affects gastrulation, and gene expression studies in the TRF3-deficient embryos further revealed that the Xenopus translation initiation factor EF1a as well as the $R b$ tumor suppressor genes are targets of TRF3 in these cells. Whereas transcription of the EF1a gene was also found to require TBP, $R b$ expression in frogs was dependent on TRF3, but not TBP. It is not clear whether the Xenopus $R b$ core promoter is a direct target of TRF3, nor is it known whether transcription of the mammalian $R b$ gene is exclusively regulated by TRF3. Nevertheless, the possibility is particularly intriguing given that the RB protein is critically required for terminal differentiation of muscle cells (De Falco et al. 2006) and many other cell types, including retina, neurons, and adipose tissue (Khidr and Chen 2006). Unlike wild-type mice, differentiated myotubes from mouse cells lacking $\mathrm{RB}\left(\mathrm{Rb}^{-/-}\right)$retain the ability to re-enter the cell cycle upon addition of serum, indicating that $\mathrm{RB}$ is required both to initiate as well as maintain the permanent cell cycle withdrawal seen in differentiated cells (De Falco et al. 2006). In muscle, RB plays both a negative role in gene expression, mediated through repression of E2F-dependent genes involved in the control of cell proliferation and survival, as well as a positive role in muscle-specific gene activation, effectively enhancing the transcriptional activity of the myogenic MyoD and MEF2 factors. RB has also been reported to repress expression of the E2F-dependent EZH2 polycomb repressor protein (Bracken et al. 2003), which could facilitate differentiation through several different mechanisms, including the derepression of MyoD and other myogenic-specific genes that are targets of EZH2 in undifferentiated muscle cells (Caretti et al. 2004). A similar role for tissue-specific TAFs in counteracting polycomb repression during terminal differentiation has also been proposed by Chen et al. (2005). As a fundamental gatekeeper of the switch from cell growth and progression through the cell cycle to terminal differentiation, exclusive regulation of the $R b$ gene by TRF3 would ensure that its expression persists even as cells undergo a global loss of TFIID.

\section{Altered P-TEFb subunit composition in terminally differentiated cells}

These findings also raise the question of whether other alternative forms of core or general transcription factors might function exclusively in post-mitotic differentiated cells. The basic activation domain of the MyoD proteins cannot be functionally substituted with that of the more generic VP16 activator, suggesting that it forms a distinctive surface designed to interact with coactivators found in differentiated cells. One of several identified targets for MyoD is a muscle-enriched form of P-TEFb (De Luca et al. 2003), the RNAPII CTD Ser2 kinase that mediates productive RNAPII transcription elongation and histone $\mathrm{H} 3 \mathrm{~K} 4$ trimethylation at many mammalian genes. In muscle, CDK9 forms a complex with CycT2a (Simone et al. 2002), rather than the CycT1 protein commonly used in rapidly proliferating cells. MyoD associates with CycT2a:CDK9, but not CycT1:CDK9 complexes, and only the CycT2a:CDK9 complex enhances MyoD transcription and promotes muscle cell differentiation (Simone et al. 2002; Giacinti et al. 2006).

Interestingly, CycT1 and CycT2a levels have also been shown to differ in late differentiated monocyte-derived macrophages (MDM). In particular, CycT1 levels rise transiently upon monocyte differentiation, but decline again in late-stage differentiated MDMs as a consequence of proteasome-dependent proteolytic destruction dictated by its C-terminal PEST domain. In contrast, CycT2 proteins, which lack the PEST domain, are constitutively expressed, and their expression persists in late-stage differentiated MDM cells, where they may functionally replace CycT1 (Liou et al. 2006). Based on the relative stability of CycT1 in various undifferentiated or transformed cells, it is tempting to speculate that differentiated cells express an E3 ligase(s), or other components of the proteolytic machinery, that selectively targets CycT1, but not СycT2, for destruction in the nondividing differentiated cells (Liou et al. 2006). By extension, it would be interesting to learn whether the different CycT/P-TEFb subunits, like some of the TBP/ TFIID components, might also show selective stability in terminally differentiated muscle cells, and whether either or both of these processes are conserved in other terminal cell differentiation programs.

\section{A major shake-up in SWI2/SNF2-like chromatin remodeling complexes upon neuronal cell differentiation}

The idea that parallel transcription networks operate in proliferating versus differentiating cells is strongly supported by a recent study from the Crabtree laboratory (Lessard et al. 2007), which examined the changes in composition and function of SWI2/SNF2-like nucleosome remodeling complexes containing the Brahma ATPase family members Brahma (BRM) or Brahma-related gene (BRG1) in proliferating neural stem and progenitor cells as they commit and differentiate to various 
neuronal cell lineages in mice. BRG1 and BRM reside in large stable BAF (BRG1/BRM-associated factor) chromatin remodeling complexes that are broadly required for mitotic cell growth and transcription control. Lessard et al. (2007) found that differentiation of proliferating neural stem/progenitor cells is accompanied by the loss of two specificity-conferring subunits, termed BAF45a and BAF53a, that are replaced in post-mitotic neurons by homologous subunits-BAF45b/c and BAF53b, respectively. By immunoblot, levels of BAF45a and BAF53a can be seen to decline precipitously by embryonic day 12-13, as large numbers of neural stem cells in the developing brain start to exit the cell cycle and differentiate, concomitant with newly induced expression of the BAF45b/c and BAF53b proteins. Remarkably, functional studies show that enforced expression of BAF45b cannot substitute for the ability of BAF45a to promote neural stem/progenitor cell proliferation, which depends on an $\mathrm{N}$-terminal C2H2-type Kruppel-like zinc finger domain that is not well conserved among the two subunits. Similarly, overexpression of the BAF45a and BAF53a subunits blocked neuronal cell differentiation, indicating that each subunit contributes to the cell-type specificity of the complex. Although the underlying mechanism is not yet understood, the implication, which likely extends to other cell types as well, is that each subunit is optimally designed to fit and function with upstream activators and core transcription machinery present in proliferating or differentiating cell types.

\section{A conserved strategy for transcription in terminally differentiated cells?}

The Deato and Tjian model provides a useful framework that can be used to study and interpret the mechanism of transcription activation in highly specialized cells, and leaves open many unresolved questions for future investigations. First, how conserved is core promoter factor exchange in the differentiation programs of distinct tissue and cell types and in embryonic cell development? How extensive is the use of alternative core factor complexes within differentiated cells, and what are the mechanistic links connecting core factor composition with cell division? How are TFR3:TAF3 complexes targeted to specific genes and TBP/TFIID to others, and why do some genes appear to require both TRF3:TAF3 and TBP? Does TRF3:TAF3 function at myogenic genes also depend on the muscle-enriched CycT2a:CDK9 P-TEFb complex, or other variant transcription subcomplexes such as the BAF remodeling complex, and how does transcription driven from these core elements differ from that of their counterparts in dividing cells? Is $R b$ also a conserved direct target of the TRF3:TAF3 complex? Most intriguingly, how does transcription of RNAPI and RNAPIII genes survive the loss of TBP in differentiated cells, and what effect does the loss of TAF subunits have on genes that require the STAGA histone acetyltransferase and TFTC complexes? And are there specific chromatin modifications that characterize and distinguish these differentiation-specific genes from those bound by the canonical core factors? Apart from the questions of transcriptional control, it remains to be seen to what extent the loss of TBP and TAF subunits is due to differentiation-induced proteolysis, as opposed to transcriptional repression, and it will be important to elaborate the mechanism underlying this dramatic switch in core factor expression. Looking forward, this new function for core promoter factors should prove a rich area for investigators seeking to unravel these additional complex layers of transcriptional specificity that control cell fate.

\section{Acknowledgments}

I thank Katherine So for her editorial assistance and acknowledge funding from the NIH, which supports research on RNAPII transcription mechanisms in our laboratory.

\section{References}

Bartfai, R., Balduf, C., Hilton, T., Rathmann, Y., Hadzhiev, Y., Tora, L., Orban, L., and Muller, F. 2004. TBP2, a vertebratespecific member of the TBP family, is required in embryonic development of zebrafish. Curr. Biol. 14: 593-598.

Bassel-Duby, R. and Olson, E.N. 2006. Signaling pathways in skeletal muscle remodeling. Annu. Rev. Biochem. 75: 19-37.

Baugh, L.R. and Hunter, C.P. 2006. MyoD, modularity, and myogenesis: Conservation of regulators and redundancy in C. elegans. Genes \& Dev. 20: 3342-3346.

Bracken, A.P., Pasini, D., Capra, M., Prosperini, E., Colli, E., and Helin, K. 2003. EZH2 is downstream of the pRB-E2F pathway, essential for proliferation and amplified in cancer. EMBO J. 22: 5323-5335.

Caretti, G., Di Padova, M., Micales, B., Lyons, G.E., and Sartorelli, V. 2004. The Polycomb Ezh2 methyltransferase regulates muscle gene expression and skeletal muscle differentiation. Genes \& Dev. 18: 2627-2638.

Chen, X., Hiller, M., Sancak, Y., and Fuller, M.T. 2005. Tissuespecific TAFs counteract Polycomb to turn on terminal differentiation. Science 310: 869-872.

Creemers, E.E., Sutherland, L.B., Oh, J., Barbosa, A.C., and Olson, E.N. 2006. Coactivation of MEF2 by the SAP domain proteins myocardin and MASTR. Mol. Cell 23: 83-96.

De Falco, G., Comes, F., and Simone, C. 2006. pRb: Master of differentiation. Coupling irreversible cell cycle withdrawal with induction of muscle-specific transcription. Oncogene 25: 5244-5249.

De Luca, A., De Falco, M., Baldi, A., and Paggi, M.G. 2003. Cyclin T: Three forms for different roles in physiological and pathological functions. J. Cell. Physiol. 194: 101-107.

Deato, M.D.E. and Tjian, R. 2007. Switching of the core transcription machinery during myogenesis. Genes \& Dev. (this issue), doi: $10.1101 /$ gad.1583407.

Gangloff, Y.G., Pointud, J.C., Thuault, S., Carre, L., Romier, C., Muratoglu, S., Brand, M., Tora, L., Couderc, J.L., and Davidson, I. 2001. The TFIID components human $\mathrm{TAF}_{\mathrm{II}} 140$ and Drosophila BIP2 (TAF 1 II 15$)$ are novel metazoan homologues of yeast $\mathrm{TAF}_{\mathrm{II}} 47$ containing a histone fold and a PHD finger. Mol. Cell. Biol. 21: 5109-5121.

Gazdag, E., Rajkovic, A., Torres-Padilla, M.E., and Tora, L. 2007. Analysis of TATA-binding protein 2 (TBP2) and TBP expression suggests different roles for the two proteins in regulation of gene expression during oogenesis and early mouse development. Reproduction 134: 51-62. 
Giacinti, C., Bagella, L., Puri, P.L., Giordano, A., and Simone, C. 2006. MyoD recruits the cdk9/cyclin T2 complex on myogenic-genes regulatory regions. J. Cell. Physiol. 206: 807-813.

Grigoryev, S., Bulynko, Y., and Popova, E. 2006. The end adjusts the means: Heterochromatin remodeling during terminal cell differentiation. Chromosome Res. 14: 53-69.

Hansen, S.K., Takada, S., Jacobson, R.H., Lis, J.T., and Tjian, R. 1997. Transcription properties of a cell type-specific TATAbinding protein, TRF. Cell 91: 71-83.

Hochheimer, A., Zhou, S., Zheng, S., Holmes, M.C., and Tjian, R. 2002. TRF2 associates with DREF and directs promoterselective gene expression in Drosophila. Nature 420: 439445.

Holmes, M.C. and Tjian, R. 2000. Promoter-selective properties of the TBP-related factor TRF1. Science 288: 867-870.

Jallow, Z., Jacobi, U.G., Weeks, D.L., Dawid, I.B., and Veenstra, G.J. 2004. Specialized and redundant roles of TBP and a vertebrate-specific TBP paralog in embryonic gene regulation in Xenopus. Proc. Nat1. Acad. Sci. 101: 13525-13530.

Khidr, L. and Chen, P.L. 2006. RB, the conductor that orchestrates life, death and differentiation. Oncogene 25: 52105219.

Lessard, J., Wu, J.I., Ranish, J.A., Wan, M., Wislow, M.M., Staahl, B.T., Wu, H., Aebersold, R., Graef, I.A., and Crabtree, G.R. 2007. An essential switch in subunit composition of a chromatin remodeling complex during neural development. Neuron 55: 201-215.

Liou, L.Y., Haaland, R.E., Herrmann, C.H., and Rice, A.P. 2006. Cyclin $\mathrm{T} 1$ but not cyclin T2a is induced by a post-transcriptional mechanism in PAMP-activated monocyte-derived macrophages. J. Leukoc. Biol. 79: 388-396.

Martianov, I., Viville, S., and Davidson, I. 2002. RNA polymerase II transcription in murine cells lacking the TATA binding protein. Science 298: 1036-1039.

Perletti, L., Kopf, E., Carre, L., and Davidson, I. 2001. Coordinate regulation of $\mathrm{RAR} \gamma 2$, TBP, and $\mathrm{TAF}_{\mathrm{II}} 135$ by targeted proteolysis during retinoic acid-induced differentiation of F9 embryonal carcinoma cells. BMC Mol. Biol. 2: 4. doi: 10.1186/1471-2199-2-4.

Persengiev, S.P., Zhu, X., Dixit, B.L., Maston, G.A., Kittler, E.L., and Green, M.R. 2003. TRF3, a TATA-box-binding proteinrelated factor, is vertebrate-specific and widely expressed. Proc. Natl. Acad. Sci. 100: 14887-14891.

Pipes, G.C., Creemers, E.E., and Olson, E.N. 2006. The myocardin family of transcriptional coactivators: Versatile regulators of cell growth, migration, and myogenesis. Genes \& Dev. 20: 1545-1556.

Rabenstein, M.D., Zhou, S., Lis, J.T., and Tjian, R. 1999. TATA box-binding protein (TBP)-related factor 2 (TRF2), a third member of the TBP family. Proc. Nat1. Acad. Sci. 96: 47914796.

Sartorelli, V. and Caretti, G. 2005. Mechanisms underlying the transcriptional regulation of skeletal myogenesis. Curr. Opin. Genet. Dev. 15: 528-535.

Shen, H., McElhinney, A.S., Cao, Y., Gao, P., Liu, J., Bronson, R., Griffin, J.D., and Wu, L. 2006. The Notch coactivator, MAML1, functions as a novel coactivator for MEF2C-mediated transcription and is required for normal myogenesis. Genes \& Dev. 20: 675-688.

Simone, C., Stiegler, P., Bagella, L., Pucci, B., Bellan, C., De Falco, G., De Luca, A., Guanti, G., Puri, P.L., and Giordano, A. 2002. Activation of MyoD-dependent transcription by cdk9/cyclin T2. Oncogene 21: 4137-4148.

Smale, S.T. and Kadonaga, J.T. 2003. The RNA polymerase II core promoter. Annu. Rev. Biochem. 72: 449-479.
Takada, S., Lis, J.T., Zhou, S., and Tjian, R. 2000. A TRF1:BRF complex directs Drosophila RNA polymerase III transcription. Cell 101: 459-469.

Teichmann, M., Wang, Z., Martinez, E., Tjernberg, A., Zhang, D., Vollmer, F., Chait, B.T., and Roeder, R.G. 1999. Human TATA-binding protein-related factor-2 (hTRF2) stably associates with hTFIIA in HeLa cells. Proc. Natl. Acad. Sci. 96: 13720-13725.

Thomas, M.C. and Chiang, C.M. 2006. The general transcription machinery and general cofactors. Crit. Rev. Biochem. Mol. Biol. 41: 105-178.

Veenstra, G.J., Weeks, D.L., and Wolffe, A.P. 2000. Distinct roles for TBP and TBP-like factor in early embryonic gene transcription in Xenopus. Science 290: 2312-2315.

Verrijzer, C.P. 2001. Transcription factor IID-Not so basal after all. Science 293: 2010-2011.

Xiao, L., Kim, M., and DeJong, J. 2006. Developmental and cell type-specific regulation of core promoter transcription factors in germ cells of frogs and mice. Brain Res. Gene Expr. Patterns 6: 409-419.

Yang, Y., Cao, J., Huang, L., Fang, H.Y., and Sheng, H.Z. 2006. Regulated expression of TATA-binding protein-related factor 3 (TRF3) during early embryogenesis. Cell Res. 16: 610621.

Zhang, D., Penttila, T.L., Morris, P.L., and Roeder, R.G. 2001. Cell- and stage-specific high-level expression of TBP-related factor 2 (TRF2) during mouse spermatogenesis. Mech. Dev. 106: 203-205. 


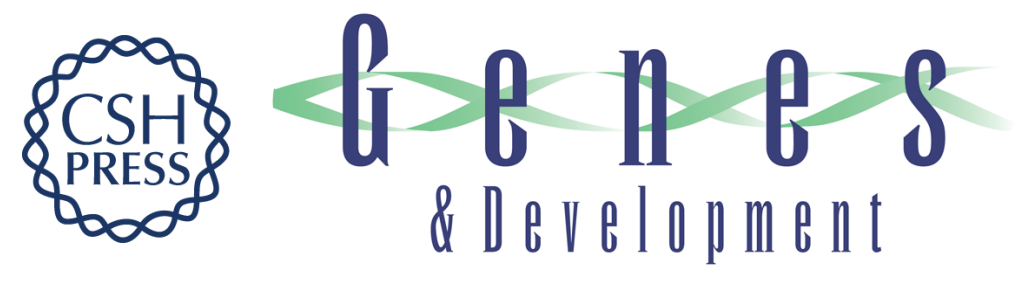

\section{Transcription strategies in terminally differentiated cells: shaken to the core}

Katherine A. Jones

Genes Dev. 2007, 21:

Access the most recent version at doi:10.1101/gad.1598007
Related Content Switching of the core transcription machinery during myogenesis
Maria Divina E. Deato and Robert Tjian
Genes Dev. September , 2007 21: 2137-2149
References This article cites 37 articles, 16 of which can be accessed free at:
http://genesdev.cshlp.org/content/21/17/2113.full.html\#ref-list-1
Articles cited in:
http://genesdev.cshlp.org/content/21/17/2113.full.html\#related-urls
License
Email Alerting
Service $\begin{aligned} & \text { Receive free email alerts when new articles cite this article - sign up in the box at the top } \\ & \text { right corner of the article or click here. }\end{aligned}$

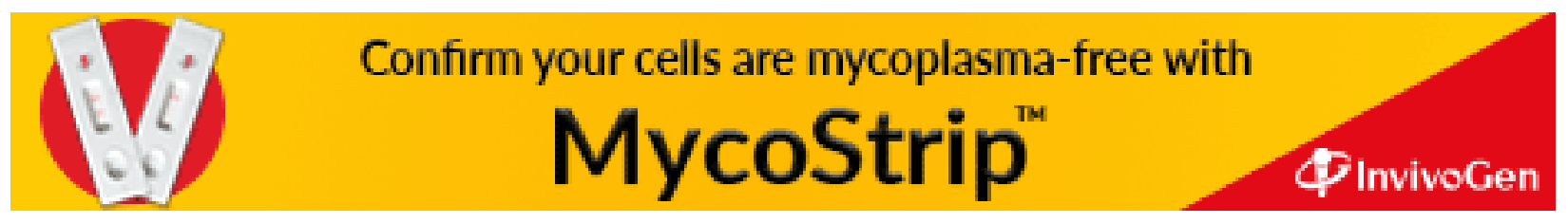

